\title{
“Agnes [...], ein seltsamer Name”. Der Name der weiblichen Protagonistin in Peter Stamms Agnes als klassifizierender Name ${ }^{1}$
}

\section{Ján Jambor}

Universität Prešov

Zusammenfassung: Der vorliegende Beitrag interpretiert einige Aspekte des titelgebenden Namens der weiblichen Protagonistin aus Peter Stamms Erstling Agnes. Roman (1998). Als Ausgangspunkt dienen dabei sowohl wissenschatliche Ansichten aus dem Bereich der literarischen Onomastik (Jurij Tynjanov, Hendrik Birus, Daniela Hodrová) als auch poetische Überlegungen einiger Autoren (Peter Stamm, Umberto Eco, Nathalie Sarraute). In Anlehnung an Hendrik Birus' Typologie der literarischen Personennamen werden zunächst vier Bedeutungsebenenen des Namens Agnes vorgestellt. Im zweiten Teil wird auf psychologische und soziokulturelle Aspekte der Namensgebung (die Wahrnehmung und die Frequenz des Vornamens) verwiesen. Anschließend wird im dritten Teil die These von der Spiegelung des seltsamen Charakters der weiblichen Hauptfigur in ihrem seltsamen Namen durch die Analyse der Passagen mit den lexikalischen Mitteln "seltsam" und "fremd" sowie mit deren Derivaten untermauert.

Schlüsselbegriffe: deutschsprachige Schweizer Gegenwartsliteratur, Peter Stamm (geb. 1963), Agnes. Roman (1998), literarische Personennamen, das Motiv des Seltsamen und des Fremden

\footnotetext{
Abstract: The given article interprets some of the aspects of the title-giving names of the female protagonist in Peter Stamm's firstling Agnes (1998). Both, academic views from the field of literary onomastic (Jurij Tynjanov, Hendrik Birus, Daniela Hodrová), as well as poetical reflections of several authors (Peter Stamm, Umberto Eco, Nathalie Sarraute), serve as the starting point. Firstly, in the style of Hendrik Birus' typology of 
literary personal names, the four levels of meaning of the name Agnes are introduced. In the second part we refer to the psychological and sociocultural aspects of the naming (the perception and the frequency of the first name). Subsequently in the third part, the assumption of reflection of the main female protagonist's odd personality in her odd name is supported by the analysis of the passages with the lexical means "seltsam" and "fremd" as well as theirs derivatives.

Keywords: contemporary Swiss German literature, Peter Stamm (born 1963), Agnes. Roman (1998), literary personal names, theme of the oddness and the strangeness

\section{Die vier Bedeutungsebenen des Namens Agnes}

$\mathrm{Zu}$ den aus Personennamen bestehenden Titeln schreibt Umberto Eco in Titel und Sinn, dem ersten Teil von Postille a Il nome della rosa (1983): "Ein Titel ist leider bereits ein Schlüssel zu einem Sinn. Niemand kann sich den Suggestionen entziehen, die von Titeln wie Rot und Schwarz oder Krieg und Frieden ausgehen. Am meisten Respekt vor dem Leser bezeugen Titel, die sich auf den Namen des Helden beschränken, wie David Copperfield oder Robinson Crusoe [...]" (Eco 1986: 10).

Im Rahmen einer bedeutenden sprachphilosophischen und linguistischen Diskussion zur Frage nach der lexikalischen Bedeutung der Eigennamen schließt sich Eco den Anhängern der These an, dass die Eigennamen keine Bedeutung, sondern nur Bezeichnungsfunktion haben. ${ }^{2}$ Möglicherweise berücksichtigt er dabei in erster Linie die im Alltag üblichen Personennamen und übersieht das Spezifikum der literarischen Personennamen. Jedoch bereits 1924 behauptete Jurij Tynjanov in Das literarische Faktum viel angemessener: "In einem Kunstwerk gibt es keine nichtssagenden Namen. Dort gibt es keine unbekannten Namen. Alle Namen sagen etwas. Jeder Name, der in einem Werk der Literatur fällt, ist bereits Bezeichnung, die in allen verfügbaren Farben glitzert. Er entfaltet mit maximaler Kraft alle Nuancen, an denen wir im Leben vorbeigehen. Für den Helden eines Literaturwerks ist 'Ivan Petrovič Ivanov' keineswegs ein farbloser Name, denn nur im Alltagsmilieu ist Farblosigkeit etwas Negatives; innerhalb einer Konstruktion wird sie 
sofort zu einem positiven Merkmal” (Tynjanov 1967: 34). Ausgehend von Charles S. Peirce setzte sich in der literarischen Onomastik die Ansicht durch, wonach sich "die Besonderheit der literarischen Eigennamen [...] als eine Verschiebung der dominant indexikalischen Nennfunktion der normalen Eigennamen in Richtung auf die symbolische Bezeichnungsfunktion des allgemeinen Wortschatzes" (Birus 1989: 39) beschreiben lässt.

In Abgrenzung zu Ecos These wird im Folgenden der aus einem Personennamen bestehende kurze Titel Agnes für einen solchen gehalten, der nicht nur "einen Schlüssel zu einem Sinn", sondern vielmehr einen ganzen Schlüsselbund $\mathrm{zu}$ mehreren Bedeutungsmöglichkeiten anbietet. Bei deren Systematisierung kann man Hendrik Birus' Vorschlag zu einer Typologie literarischer Namen (1987) sinnvoll anwenden. Mit Hilfe der binären Opposition "Kontiguität - Similarität", "nicht-einzelsprachlich - einzelsprachlich", "individuelle Semantisierung - serielle Semantisierung" unterscheidet Birus vier Namentypen, und zwar verkörperte, klassifizierende, klangsymbolische und redende Namen (vgl. idem: 45) wobei er auf die Möglichkeit von Mischformen verweist (vgl. idem: 47-48).

Beim literarischen Namen Agnes handelt es sich primär um eine Mischform von verkörpertem und redendem Namen, für die individuelle Semantisierung und klare Charakterisierungsfunktion typisch sind (vgl. idem: 46). Diese zwei Namentypen fasst Birus "auf Grund ihrer funktionellen oder genetischen Affinität zu den Appellativa als Appellativnamen" (idem: 46) zusammen. Die restlichen zwei Namentypen (klassifizierende und klangsymbolische Namen), “deren Bedeutungsfärbung zumeist in Analogie oder Kontrast mit dem übrigen Namenensemble einer Dichtung oder aber auf der Basis von 'Appellativnamen' zur Abhebung kommt", fasst Birus als "suggestive Namen" (beides idem: 47) zusammen. Wie später gezeigt wird, sind diese zwei Namentypen - im Kontext der anderen Figuren des Romans und der Figuren aus Stamms anderen Texten - auch beim Titel Agnes zu berücksichtigen.

In Anlehnung an die vier Namentypen kann man vier Bedeutungsebenen des Titels Agnes unterscheiden. Die erste Bedeutungsebene lässt sich durch die Interpretation der intertextuellen Bezüge des Titels erschließen. Nach Birus sind die verkörperten Namen 
"literarische Eigennamen, deren Semantisierung vornehmlich auf der Kontiguitätsassoziation eines bereits (real oder fiktional) existierenden Trägers dieses Namens und dessen Eigenschaften beruht [...]" (idem: 45). Dies ist auch bei Stamms Erstling der Fall, dessen Titel in erster Linie auf den Stoff um die Heilige Agnes von Rom, eine frühchristliche Märtyrerin der spätantiken Welt, verweist. Von verschiedenen Literarisierungen dieses Stoffes sind für Stamms Agnes zwei Intertexte von zentraler Relevanz. Einerseits handelt es sich um das einschlägige Kapitel zur genannten Märtyrerin in Jacobus de Voragines lateinischer Legenda aurea (etwa zwischen 1263 und 1273 entstanden). Andererseits handelt es sich um John Keats' romantische Verserzählung The Eve of St. Agnes (1820), aus der Stamm zwei Verse zum Motto seines Erstlings wählte (vgl. A: o. S.). ${ }^{3}$

Die zweite Bedeutungsebene des Titels Agnes lässt sich unter Berücksichtigung der Etymologie des titelgebenden Namens erschließen, dessen "Semantisierung vornehmlich auf der [...] Similaritätsassoziation von Elementen des allgemeinen Wortschatzes beruht" (Birus 1987: 45), einem wesentlichen Charakteristikum der redenden Namen. Da die Herkunft des Namens Agnes mit der Geschichte der Heiligen Agnes von Rom in Verbindung steht, ${ }^{4}$ sind die beiden Bedeutungsebenen unauflöslich miteinander verknüpft.

Bei der Erschließung der dritten Bedeutungsebene kann man sich auf die zwei einzigen Textstellen stützen, in denen die Problematik des Namens Agnes reflektiert wird. Wie im nächsten Teil gezeigt wird, verweisen sie auf soziokulturelle Aspekte der Namensgebung (die Frequenz und die Wahrnehmung des Namens der weiblichen Protagonistin). Dadurch nähert sich der Name Agnes den klassifizierenden Namen, “deren Semantisierung vornehmlich auf der Kontiguitätsassoziation bestimmter (nationaler, religiöser, sozialer u. a.) Gruppen von Namensträgern beruht, die durch den allgemeinen Sprachgebrauch oder aber durch feste literarische Konventionen bedingt ist [...]" (Birus 1987: 45).

Schließlich gibt es auch einen möglichen, wenn auch nur partiellen Anschluss zu den klangsymbolischen Namen, "deren Semantisierung vornehmlich auf ihren ikonischen Qualitäten (z. B. onomatopoetischen, synästhetischen, diagrammatischen 
Similaritätsassoziationen) beruht [...]" (ibidem) In den Materialien zu Agnes, die Stamm im Februar 2012 in erster Linie für Schüler und Lehrer auf seiner Homepage zur Verfügung gestellt hat, steht: "Bevor meine Figuren Namen haben, bezeichne ich sie in meinen Notizen oft mit zufälligen Initialen. Die Männerfiguren bezeichne ich meistens mit 'A.' wie Adam. (Und oft tragen sie später auch in den Büchern Namen, die mit A beginnen, wie Andreas in 'An einem Tag wie diesem' oder Alexander in 'Sieben Jahre')" (Stamm 2012: o. S.) Stamms Vergleich der Initiale "A.", des ersten Buchstaben des lateinischen Alphabets, mit "Adam”, dem Namen des ersten Menschen nach der biblischen Überlieferung, kann als Hinweis auf den "Anthropos", den Menschen allgemein verstanden werden. Im letztgenannten Zitat ist ausdrücklich von den männlichen Figuren die Rede und aus der Entstehungsgeschichte des Textes geht klar hervor, dass die weibliche Protagonistin des Erstlings zunächst als kein “A.", sondern als "ein Mädchen” bezeichnet wurde (vgl. ibidem u. Stamm 2014: 74). Trotz dieser zwei Tatsachen kann man die These von der anthropologischen Universalität auch auf die Figur Agnes anwenden. Das am Beginn des Namens der weiblichen Protagonistin und am Anfang des Titels stehende "A" ist mit Hilfe der klangsymbolischen Assoziation als ein Hinweis auf die anthropozentrische Orientierung zu verstehen, die für Stamms Texte typisch ist. Seine Antwort auf die von Olga Olivia Kasaty gestellte Frage, worüber er schreibe, eröffnet Stamm mit einem treffenden Satz: "Ich schreibe über Menschen und über Beziehungen zwischen Menschen" (Kasaty/ Stamm 2007: 395).

\section{Die Frequenz und die Wahrnehmung des Namens Agnes}

Wie angedeutet, gibt es in Stamms Erstling nur zwei Textstellen, in denen die Problematik des Namens Agnes reflektiert wird. Die erste Textstelle steht am Ende des ersten Treffens der beiden Protagonisten. Der Ich-Erzähler beobachtet im Lesesaal der Chicago Public Library längere Zeit eine ihm gegenüber sitzende unbekannte Frau, deren Äußeres "nicht auffallend" ist und die ihn trotzdem "interessierte" und "beschäftigte" (alles A: 2, 14), wie er zugibt. Bei ihrem ersten Gespräch, während sie auf der breiten Freitreppe vor dem Eingang in das Gebäude sitzend rauchen, stellen sie sich seltsamerweise nicht gleich vor, sondern unterhalten sich belanglos “über die Bibliothek, die Stadt, das Wetter" 
(A: 2, 15). Erst als sie aufstehen, um in den Lesesaal zurückzukehren, fragt er sie "nach ihrem Namen. Sie sagte, sie heiße Agnes. 'Agnes', sagte ich, 'ein seltsamer Name.' 'Sie sind nicht der erste, der das sagt'“ $(A: 2,16)$.

Aus den Zitaten geht hervor, dass der Vorname der Protagonistin im Kontrast zu ihrem äußeren Erscheinungssbild steht. Zum einen reagiert der Ich-Erzähler auf den Vornamen mit der Bemerkung über dessen Seltsamkeit, zum anderen reiht die Protagonistin den vor kurzem kennengelernten Mann in eine Reihe mit einer nicht genau definierten Gruppe von Personen, die gleicher Meinung sind. Da Agnes in Chicago aufgewachsen ist (vgl. A: 3, 20) und lebt, ist wenigstens anzunehmen, dass es sich dabei zumeist um US-Amerikaner handelt. Nicht genau definiert sind auch die Einstellung der Protagonistin zu ihrem eigenen Namen und was genau mit dem Adjektiv "seltsam" gemeint ist. Ein Name kann als seltsam aufgrund von seinem seltenen Vorkommen wirken, ferner aufgrund von seinen lautlichen Qualitäten oder aber auch aufgrund von semantischen Assoziationen, die er hervorruft.

Die zweite Textstelle, in der die Problematik des Namens Agnes reflektiert wird, beinhaltet die Reaktion von Louise, als sie den Namen ihrer Rivalin zum ersten Mal hört: “Ich erzählte Louise von Agnes und daß sie mich verlassen habe. [...] 'Agnes', sagte sie. 'Ein lustiger Name. War das deine kleine Freundin, die Amerikanerin mit der Wollunterwäsche?” (A: 21, 97-98).

Ähnlich wie bei dem Adjektiv "seltsam" ist auch die Bedeutung des Adjektivs "lustig" nicht eindeutig klar. Es ist denkbar, dass Louise den Namen für lustig hält, weil er selten ist, altmodisch klingt oder konservativ bzw. katholisch geprägt ist. Unter Berücksichtigung der ganzen kommunikativen Situation und der für Louise typischen Eigenschaften ist recht deutlich, dass bei der herabsetzen Abqualifizierung des Namens Louises Eifersucht auf Agnes genauso eine wichtige Rolle spielt wie ihr Zynismus, Spott und Ironie.

Beide Bezeichnungen des Namens ("seltsam" und "lustig") sind ein Resultat von subjektiv geprägten Wahrnehmungen. Da sie im Text nicht näher erklärt werden, sind sie für den Leser nicht eindeutig interpretierbar. Beide sind allerdings durch die Seltenheit des 
Namens Agnes bedingt. Im Folgenden wird die Aufmerksamkeit auf diesen objektiven Faktor gelenkt.

Der Information der Sektion Demografie und Migration bei dem Bundesamt für Statistik in Neuchâtel zufolge stehen leider keine genauen Zahlen zur Verfügung, wieviele Personen einen konkreten Namen in der Schweiz der 90er Jahre trugen bzw. tragen (vgl. Grisati Favre, 30. 07. 2012, o. S.). Die einzelnen vorhandenen Zahlen zur Frequenz der Vornamen in der Schweiz stammen aus der Statistik der natürlichen Bevölkerungbewegung (BEVNAT). Sie geben an, wieviele neugeborene Personen in der Schweiz in einem bestimmten Jahr einen konkreten Namen erhalten haben (vgl. ibidem). ${ }^{5}$ Die per E-Mail als Anhang auf Anfrage zugeschicken Excel-Tabellen des Bundesamtes für Statistik, gegliedert nach sprachlichen Regionen der Schweiz, bestätigen, dass sich der weibliche Vorname Agnes keiner großen Beliebtheit bei den Eltern in der Schweiz der 90er Jahre erfreute. 1992, ein Jahr vor der Entstehung der ersten Fassung von Agnes, wurden in der deutschsprachigen Schweiz fünf Mädchen auf diesen Namen getauft. Dieselbe Zahl finden wir auch im darauffolgenden Jahr, allerdings bei der slawisch anmutenden Variante Agnesa. In der Zeit, als Stamm die Arbeit am Roman wiederaufgenommen hat, waren es 1996 bei dieser Variante neun, 1997 dreizehn und 1998 zehn Kinder. Auch in der Romandie ist die Situation nicht anders. 1993 und 1996 erhielten je acht weibliche Personen den Vornamen Agnes, 1997 und 1998 keine. In der Tabelle zur italienisch- und rätoromanischsprachigen Schweiz, die - wie die vorherigen zwei die Zahlen seit 1987 verzeichnet - kommt der Vorname Agnes oder seine Varianten bis Ende der 90er Jahre gar nicht vor (vgl. Grisati Favre, 27. 07. 2012, o. S.).

In den USA ist ein deutlicher Abstieg der Popularität des Vormanens Agnes zu beobachten: "It remained a widely used name throughout the 1960s in the United States. It was last ranked among the top 1000 names for American girls during that decade. The peak of its popularity was between 1900 and 1920, when it was among the top 50 names for American girls" (O. A., Agnes (name): o.S.) Den Angaben der Statistik 1000 Most Common Female Names in the U.S. zufolge, die das englischsprachige Online-Magazin Mongabay publizierte, belegt der Name Agnes aktuell den Platz 215 - 218 unter den weiblichen 
Vornamen in den USA. Genauso wie bei den Namen Jessie, Natalie und Vera ist seine Frequenz 0,098 \% und sein "Aproximate number" 148773 (vgl. o. A., 1000 Most Common Female Names in the U. S.: o. S.).

Obwohl die genauen Zahlen betreffend aller in der Schweiz lebenden Personen fehlen und obwohl die Untersuchungssmethoden zur Erhebeung der Zahlen in den USA nicht näher bekannt sind, ist es anzunehmen, dass der Name Agnes in den beiden Ländern eher selten ist.

\section{Der seltsame Name als Spiegelung des seltsamen Charakters}

Wichtiger als die Angaben zur Frequenz des Namens sind die nachfolgenden Überlegungen zur Funktion von seltsamen (ungewöhnlichen) Namen in literarischen Texten. Nach Hodrová war v. a. in der Literatur des Realismus der gewöhnliche Name ein Zeichen für eine gewöhnliche Figur und der ungewöhnliche Name ein Zeichen für eine ungewöhnliche Figur (vgl. Hodrová et al. 2001: 604). Der Name in solchen Texten des 19. Jahrhunderts machte zumeist nicht auf sich selbst aufmerksam. Er war nicht auffallend und demonstrierte daher nicht seinen fiktiven Charakter (vgl. idem: 610).

Die gezogene Parallele zwischen (un)gewöhnlichen Figuren und (un)gewöhnlichen Namen gilt zweifelsohne nicht nur für die realistische Literatur. Ein gutes Beispiel dafür ist auch Stamms Protagonistin, deren seltsamer Name mit ihrem seltsamen Charakter korrespondiert. Auf diese Tatsache weist Birgid Schmid hin, indem sie zur oben zitierten Aussage des Ich-Erzählers, der Name Agnes sei seltsam, schreibt: "Das ist das erste Indiz im Roman, dass die Namensträgerin einen sonderbaren Charakter hat - Einzigartigkeit erlangt Agnes also gerade durch den sie bezeichnenden Namen. Der Eigenname etikettiert die Figur als Handlungsträgerin mit einer bestimmten Funktion [...]: Geheimnisaura zu verbreiten" (Schmid 2004: 108).

Gegen das Zitat ist zweierlei einzuwenden. Erstens ist die am Ende des Kapitels 2 geäußerte Aussage des Ich-Erzählers sicherlich ein wichitger Hinweis auf Agnes' sonderbaren Charakter, aber bestimmt ist es nicht das erste Indiz dafür. Bereits am Ende des Kapitels 1 beschreibt der Ich-Erzähler die Angstgefühle seiner Partnerin, die etwas von 
ihrem sonderbaren Charakter vermuten lassen. Agnes hat Angst vor Personen und Sachverhalten, die üblicherweise einem Menschen (z. B. dem Ich-Erzähler, der bei solchen Situationen anwesend ist) keine Angst bereiten - vor dem Verkäufer im Laden im Doral Plaza, vor nicht zu öffnenden Fenstern im Wolkenkratzer, vor nächtlichem Summen der Klimaanlage und vor den in einer Gondel schwebenden Fensterputzern, die Agnes vor dem Schlafzimmerfenster beobachtet (vgl. A: 1, 11-12). Darüber hinaus weist der Ich-Erzähler bei der einleitenden Beschreibung des äußeren Erscheinungsbildes von Agnes auf ein Detail hin, das deutlich erkennen lässt, dass der seltsame Vorname der Protagonistin nicht nur als Kontrast, sondern auch als Komplement zu ihrem Äußeren angelegt ist: "Nur ihr Blick war außergewöhnlich, als könne sie mit den Augen Worte übermitteln” (A: 2, 14). Zweitens kann nicht behauptet werden, dass Agnes ihre Einzigartigkeit durch den sie bezeichnenenden Namen erlangt. Bei einer solchen nominalistischen These, nach der ein Name die Eigenschaften einer literarischen Figur determinieren soll, handelt es sich um einen logischen Fehler. Bei der Textproduktion funktioniert die Namensgebung eigentlich umgekehrt, d. h. der Name einer Figur kann ihre Eigenschaften spiegeln oder etwas von ihrem Wesen andeuten.

Eine detaillierte Analyse des seltsamen Charakters von Agnes, der sich in ihrer Eigenartigkeit, Außergewöhnlichkei, ja Rätselhaftigkeit zeigt, würde den Rahmen der vorliegenden Untersuchung sprengen. Im Folgenden wird nur darauf eingegangen, dass der Ich-Erzähler in verschiedenen Situationen und Phasen der Liebesbeziehung in Bezug auf Agnes wiederholt das Wort "seltsam" bzw. das semantisch nahe stehende Wort "fremd" und seine Derivate verwendet. Die Funktion dieser Motive besteht darin, die für Agnes zentrale Problematik der Andersartigkeit in ihren verschiedenen Facetten (z. B. Geschlechterdifferenz und andere Genderaspekte, Kultur- und Mentalitätsunterschiede, psychologisch erklärbare Unterschiede im Charakter einzelner Figuren) zu entfalten.

Nachdem der Ich-Erzähler den Namen Agnes als seltsam bezeichnet hat, erzählt ihm dessen Trägerin bei ihrem zweiten Treffen am folgenden Tag von einer Begegnung ihres Freundes Herbert mit einer Frau. Die Nacherzählung des rätselhaften Treffens versieht der Ich-Erzähler mit einem Rahmen, den das Adjektiv “seltsam” bildet. Agnes' Nacherzählung 
der Geschichte leitet er mit dem Urteil ein, es handle sich um "ein seltsames Erlebnis" (A: 3, 18), und als Agnes zu Ende erzählt hat, nennt er die Episode “[e]ine seltsame Geschichte” (A: 3, 19). Laut Agnes habe sich Herbert beim Erblicken der namentlich nicht bekannten Frau "ganz seltsam gefühlt. [...] Es sei ihm gewesen, als kenne er die Frau. Dabei war er sicher gewesen, sie noch nie zuvor gesehen zu haben" $(A: 3,18)$. Während die Feststellung des Ich-Erzählers, der Name Agnes sei seltsam, für den Leser eine vorausdeutende Funktion erfüllt, ist die von Agnes erzählte Geschichte als Spiegel zum ersten Treffen zwischen ihr und dem Ich-Erzähler angelegt. Agnes deutet damit dem Ich-Erzähler zum einen an, dass sie - wie damals Herbert - eine ähnliche, nicht rational fassbare Faszination von einer Person anderen Geschlechts erlebt. Zum anderen gibt sie dem Ich-Erzähler einen Fingerzeig, dass er ihr genauso seltsam wie sie ihm vorkommt.

Zweitens wird Agnes' äußeres Erscheinungsbild bzw. Verhalten vom Ich-Erzähler stellenweise für seltsam gehalten. Bereits beim ersten Besuch eines Lokals am dritten Tag der gemeinsamen Bekanntschaft berichtet der Ich-Erzähler: "Wenn wir diskutierten, lag in allem, was Agnes sagte, ein seltsamer Ernst, ihre Ansichten waren streng" (A: 3, 21). Agnes spricht kein Deutsch, zeigt jedoch Interesse an der Schriftsprache ihres Freundes (vgl. A: 6, 30). Beim ersten Besuch von Agnes' Wohnung wird ersichtlich, dass die US-Amerikanerin eine besondere Vorliebe für die bildende Kunst des Expressionismus hat. Neben einer "Gebirgslandschaft von Ludwig Kirchner" hängt in ihrem Zimmer Oskar Kokoschkas Theaterplakat Mörder, Hoffnung der Frauen, dessen Titel sie zum Ich-Erzähler in der Originalsprache sagt. Ihr Partner kommentiert dies wie folgt: "[...] es war seltsam, sie in meiner Sprache sprechen zu hören. Ihre Stimme wirkte anders als sonst, rauher und älter." (beides A: 8, 39).

Später, als der Ich-Erzähler im Septemer beim Schreiben der Geschichte "die Gegenwart überholt" hat und zu wissen glaubt, "was geschehen wird" (A: 12, 62), beugt sich Agnes seinem Diktat. Der Ich-Erzähler gibt ihr zu verstehen, dass er einen wichtigen Entschluss im Hinblick auf ihre Beziehung gefasst und in der Geschichte verschriftlicht hat. Agnes ist neugierig, will jedoch nicht selbstständig handeln: "Erst will ich wissen, was ich zu tun habe [...], ich möchte keine Fehler machen." Von seinem Platz aus kann der Ich-Erzähler 
das Gesicht seiner Partnerin nicht sehen, aber er stellt fest: "Ihre Stimme klang seltsam kühl." (beides A: 13, 65).

Und drittens, als Agnes im Oktober bei der Wanderung im Hoosier National Forest in Ohnmacht fällt, bemerkt der Ich-Erzähler "Mit seltsam verrenkten Beinen lag sie auf dem feuchten Sand" (A: 15, 73). Zusammen mit anderen körperlichen Motiven (z. B. kreidebleiche Lippen, schwacher Herzschlag, feuchte und kalte Stirn) wird die Todesgefahr assoziiert. Diese steht im Kontrast zum wahren Grund für physische Schwäche der weiblichen Protagonistin, der nicht mit dem Sterben, sondern mit der Geburt verbunden ist. Agnes macht nämlich in einer symbolischen Zeit - die Wanderung im Nationalpark findet am verlängerten Wochenende zum Columbus Day statt - die Entdeckung, dass sie möglicherweise schwanger ist.

Bedeutender als die zitierten disparaten Textstellen, in denen das Wort "seltsam" allerdings keinesfalls zufällig verwendet wird, ist das Kapitel 11. Darin wird der Ausflug an einen See geschildert, den das Paar Anfang September unternimmt. ${ }^{6}$ Der Angabe des IchErzählers zufolge wirkt Agnes bereits zu Beginn des Ausflugs "seltsam fremd." Sie wartet auf ihren Partner sitzend "in einem der schwarzen Ledersessel" in der Eingangshalle des Doral Plaza. Der Ich-Erzähler schreibt diesen seltsamen Eindruck der ungewöhnlichen Kleidung ("dunkelblaue Knickerbocker, ein weißes T-Shirt") und schweren Schuhen zu, "die aussahen, als seien sie noch nie getragen worden." (alles A: 11, 57) Später in der Natur sagt er Agnes, "sie sehe heute anders aus als sonst [...]" (A: 11, 58). Ihrer Meinung nach hängt dies mit der neuen Frisur zusammen, was der Ich-Erzähler bestreitet (vgl. A: 11, 58). Seine Erklärung des Phänomens präsentiert er dem Leser später, als er die auf einer kleinen Lichtung in der Nachmittagssonne schlafende Agnes beobachtet. Ihr Gesicht erscheint ihm “wie eine unbekannte Landschaft." Nach einer Passage, in der einzelne Teile von Agnes' Gesicht mit Naturphänomenen verglichen werden, kommt der Partner zum Schluss: "Das ganze Gesicht schien mir fremd, unheimlich, und doch war es mir, als sähe ich es wirklicher als jemals zuvor, unmittelbar. Obwohl ich Agnes nicht berührte, hatte ich das beängstigende und zugleich berauschend schöne Gefühl, sie wie eine zweite Haut einzuhüllen, ihren ganzen Körper auf einmal dicht an mir zu spüren.” Als Agnes erwacht, reagiert ihr Partner 
eher mit Körpersprache und Schweigen denn mit Worten und im Gespräch beschränkt er sich nur auf die Erklärung "Ich hatte ein seltsames Gefühl [...], daß ich dir ganz nahe sei." (alles A: 11, 58-59).

Das seltsame Gefühl verliert sich aus der Beziehung infolge der negativen Ereignisse (die Trennung des Paars, die erlittene Fehlgeburt). Nach der Wiederaufnahme der Beziehung protokolliert der Ich-Erzähler: "Langsam schien Agnes sich zu erholen. Aber es war, als habe sie sich von mir entfernt, als suche oder finde sie die Nähe zu mir nicht mehr" (A: 28, 121). Zu Weihnachten spricht er sogar vom “Gefühl der Fremdheit zwischen Agnes und mir" (A: 31, 135). Dadurch verliert Agnes ihre Sonderstellung im Leben des Protagonisten. Man sieht keinen erheblichen Unterschied zwischen dieser Beziehung und der Beziehung des Ich-Erzählers zum Ladenverkäufer im Doral Plaza, der ihm "so fremd" (A: 34, 149) ist, wie die Leute, die er im Foyer des Wolkenkratzers seit einem Jahr zufällig sieht.

Von den Motiven der Fremdheit und Seltsamkeit ist schließlich auch das erste Kapitel des Romans beherrscht, das zusammen mit dem letzten Kapitel einen zeitlichen Rahmen zur in den Kapiteln 2 bis 35 präsentierten Vergangenheitshandlung bildet. Beide Kapitel spielen sich am Neujahrstag ab, nachdem Agnes ihren Partner verlassen hat. Der Ich-Erzähler befindet sich in seiner Wohnung, die ihm "jetzt, wo Agnes gegangen ist, [...] fremd und unerträglich geworden" (A: 1, 9) ist. Er schaut sich wiederholt das von Agnes aufgenommene Video "Columbus Day in Hoosier National Forest" (A: 1, 10) an. Da Agnes auch den anscheinend schlafenden Ich-Erzähler gefilmt hat und er nun die gesehenen Bilder beschreibt, handelt es sich dabei um ein medial-narratives Spiegelbild zur oben zitierten Beschreibung der schlafenden Agnes aus dem Kapitel 11. Stamm lässt konsequent nur Agnes' stummes Produkt (das aufgenommene Video), nicht aber Agnes' Inneres (z. B. ihre Beweggründe, den Partner aufzunehmen, ihre Gefühle oder Meinung dabei) zur Sprache kommen. Die Obsession, mit der der Partner aufgenommen wurde, deutet allerdings darauf hin, dass Agnes ihn seltsam fand bzw. von ihm fasziniert war: "Ich liege auf dem Boden, scheine zu schlafen oder habe zumindest die Augen geschlossen. Die Kamera nähert sich mir von oben, kommt immer näher, bis das Bild unscharf wird, weicht 
zurück. Dann wandert sie über meinen Körper, bis zu den Füßen und wieder zum Kopf. Lange bleibt sie auf dem Gesicht stehen, versucht, noch einmal näher zu kommen, aber das Bild wird wieder unscharf, und sie weicht von neuem zurück" $(A: 1,11)$.

Agnes' Inneres bleibt für den Leser, genauso auch für den Ich-Erzähler, ein Rätsel. Von der Frau, die den Mann verlassen hat, bleibt ein Video zurück, das der Mann zu interpretieren versucht: "Manchmal erahne ich, weshalb Agnes die Kamera eingeschaltet hat: eine seltsam geformte Wolke, eine Reklametafel, in der Ferne ein Streifen Wald, fast unsichtbar durch das Weitwinkelobjektiv" (A: 1, 10). Doch das Objekt der Untersuchung entzieht sich seinen interpretatorischen Versuchen. Die wiederholt angeschauten Bilder des Videos hnterlassen bei dem Ich-Erzähler nur eine Essenz von Agnes, das Gefühl der Seltsamkeit: "Ich habe den Ton des Fernsehers ausgeschaltet. Die Bilder scheinen mir wirklicher als die dunkle Wohnung, die mich umgibt. Es ist ein seltsames Gefühl in ihnen, das Licht einer weiten Ebene an einem Nachmittag im Oktober" (A: 1, 10). ${ }^{7}$

Die Analyse der Passagen mit den lexikalischen Mitteln "seltsam" und "fremd" sowie mit ihren Derivaten zeigt, dass sie nicht nur der Entfaltung der Problematik der Andersartigkeit dienen. Diese Mittel umkreisen zugleich eine andere Problematik, für die Stamm eine besondere Vorliebe hat, nämlich dienige der Distanz und Nähe in den zwischenmenschlichen Beziehungen. Seine Antwort auf Kasatys Frage, worüber er schreibe, schließt Stamm wie folgt ab: "Dann interessieren mich Liebesbeziehungen, ganz einfach, weil es da eine fast unbeschränkte Anzahl unterschiedlicher Formen und Dynamiken gibt. Und von der Liebe kommt man ganz automatisch auf die Unmöglichkeit der Liebe. Also auf die Unmöglichkeit, dass zwei Menschen eins werden. Distanz und Nähe sind Themen, die mich interessieren" (Kasaty/ Stamm 2007: 396).

Zum Schluss sei noch auf eine andere Funktion des seltsamen Namens Agnes verwiesen. Bei den Autoren, die den fiktiven Charakter des Textes unterstreichen wollen, werden eher die seltsamen Namen bevorzugt. So schreibt z. B. Nathalie Sarraute in ihrem Essay L'Ère du soupçon (erstveröffentlicht in der Zeitschrift Les Temps modernes im Februar 1950) über ihren Kollegen André Gide: “Gide évite pour ses personnages les noms patronymiques qui risquent de les planter d'emblée solidement dans un univers trop 
semblable à celui du lecteur, et préfère les prénoms peu usuels [...]" (Sarraute 1996: 1585). Dies gilt auch für Agnes, die Protagonistin eines Textes, dessen Autor zugibt, dass es in seinen Werken Themen gibt, "die immer wieder auftauchen, zum Beispiel das Verhältnis von Realität und Fiktion, von Bild und Wirklichkeit” (Kasaty/ Stamm 2007: 396).

\section{Bibliographie}

Birus, Hendrik (1987), "Vorschlag zu einer Typologie literarischer Namen”, LiLi. Zeitschrift für Literaturwissenschaft und Linguistik, 17 (1987), H. 67: Namen. S. 38-51.

Blanár, Vincent (1996), Teória vlastného mena. Status, organizácia a fungovanie v spoločenskej komunikácii, Bratislava,Veda.

Eco, Umberto (1986), Nachschrift zum “Namen der Rose”. Aus dem Italienischen übersetzt von Burkhart Kroeber, München - Wien, Hanser [1983].

Grisafi Favr, Elena (27. 7. 2012), E-Mail-Schreiben an Ján Jambor, o. S. [Nicht veröffentlicht.] -- (30. 7. 2012), E-Mail-Schreiben an Ján Jambor, o. S. [Nicht veröffentlicht.]

Hodrová, Daniela et al. (2001), ... na okraji chaosu... Poetika literárního díla 20. století, Praha, Torst.

Hoorn, Tanja van (2010), "Peter Stamm”, in Heinz-Ludwig Arnold (ed.), Kritisches Lexikon zur deutschsprachigen Gegenwartsliteratur, 96. Nlg, 10/2010. S. 1-10 u. A-G.

Jambor, Ján (2012): “Zur Macht der Fiktion im Bereich der alltäglichen Vorstellungskraft in Peter Stamms 'Agnes'“, in Gonçalo Vilas-Boas / Teresa Martins de Oliveira (eds.), Macht in der Deutschschweizer Literatur. Berlin, Frank \& Timme, S. 335-351. 
Kasaty, Olga Olivia / Stamm, Peter (2007), "Ein Gespräch mit Peter Stamm. Krakau, 24. April 2004", in Olga Olivia Kasaty, Entgrenzungen. Vierzehn Autorengespräche, München, edition text + kritik im Richard Boorberg Verlag, S. 395-430.

O. A., Agnes (name) (o. J.), https://en.wikipedia.org/wiki/Agnes \%28name\%29 \#References. [Seite besucht am 8. 2. 2016.]

O. A., 1000 Most Common Female Names in the U. S. (o. J.), http://names.mongabay.com/female names.htm. [Seite besucht am 8. 2. 2016.]

O. A., Erhebungen, Quellen - Statistik der natürlichen Bevölkerungsbewegung (BEVNAT). Steckbrief (o. J.), http://www.bfs.admin.ch/bfs/portal/de/index/infothek/ erhebungen_quellen/blank/blank/bevnat/01.html. [Seite besucht am 15. 8. 2012.]

Sarraute, Nathalie (1996), OEuvres complètes. Édition publiée sous la direction de Jean-Yves Tadié, Paris, Éditions Gallimard.

Seibicke, Wilfried (1996), Historisches Deutsches Vornamenbuch, Bd. 1. A - E. Berlin - New York, de Gruyter..

Schmid, Birgit (2004), Die literarische Identität des Drehbuchs. Untersucht am Fallbeispiel "Agnes" von Peter Stamm, Bern - Berlin - Bruxelles - Frankfurt am Main - New York Oxford - Wien, Lang.

Stamm, Peter (1998), Agnes. Roman, Zürich - Hamburg, Arche.

-- (2012), Agnes. Quellen, http://www.peterstamm.ch/quellen.php. [Seite besucht am 27. 11. 2015 ]

-- (2014), Die Vertreibung aus dem Paradies. Bamberger Vorlesungen und verstreute Texte, Frankfurt am Main, Fischer.

Tynjanov, Jurij (1967), Die literarischen Kunstmittel und die Evolution in der Literatur. Aus dem Russischen übersetzt von Alexander Kaempfe, Frankfurt am Main, Suhrkamp.

Voragine, Jacobus de (1982), Legenda aurea. Heiligenlegenden. Auswahl, Übersetzung aus dem Lateinischen, Anmerkungen und Nachwort von Jacques Laager, Zürich, Manesse. 
Mgr. Ján Jambor, PhD., Studium der Germanistik und Slowakistik, Mitarbeiter des Instituts für Germanistik, Philosophische Fakultät, Universität Prešov, Slowakei. Schwerpunkte in Forschung, Lehre und Übersetzung: deutschsprachige Schweizer Literatur vom ausgehenden 19. Jh. bis zur Gegenwart, Theorie und Geschichte der Kriminalliteratur, Transtextualität und Intermedialität. Monographie Die Rolle des Zufalls bei der Variation der klassischen epischen Kriminalliteratur in den Bärlach-Romanen Friedrich Dürrenmatts (2007). Lehrwerk Im Umfeld der Neuen Subjektivität. Die Erzählprosa der Bundesrepublik Deutschland der 70er Jahre (2014). Übersetzung von Peter Stamms Agnes (2006), Ungefähre Landschaft (2010), Blitzeis. Hörspiel (2010), Warum wir vor der Stadt wohnen (2014), An einem Tag wie diesem (im Druck) und von Hansjörg Schneiders Hunkeler macht Sachen (2012) Aktuelle Projekte: Habilitationsschrift Die autographen Paratexte in Peter Stamms "Agnes". Titel, Untertitel, Widmung und Motto im Kontext der Intertetualität und der Architextualität, Übersetzung von Hanna Johansens Ich bin hier bloß die Katze

\section{Fussnoten}

${ }^{1}$ Der Beitrag ist im Rahmen des Forschungsprojekts VEGA 2/0063/16 Hyperlexikón literárnovedných pojmov a kategórií II (Hyperlexikon literaturwissenschaftlicher Begriffe und Kategorien II) entstanden.

2 Die sprachphilosphischen Beiträhr zu dieser Diskussion sind im von Ursula Wolf herausgegebenen Band Eigennamen. Dokumentation einer Kontroverse (1985) gesammelt. Im Unterschied zur Sprachphilosophie, in der zwei extreme Ansichten (Eigennamen haben keine Bedeutung versus Eigennamen verfügen über das höchste Maß an Bedeutung) überwiegen, sucht die Linguistik nach einem dritten Weg, wonach Eigennamem durch semantische Reduktion gekennzeichnet sind. Vgl. Blanár 1996: 52-57.

${ }^{3}$ Im Folgenden wird Agnes als A mit Kapitel- und Seitenzahl zitiert.

N. ${ }^{\circ} 34$ - 06/ 2016 | 431-447 - ISSN 1645-1112 | http:/dx.doi.org/10.21747/16451112/litcomp34a26 INSTITUTO DE LITERATURA COMPARADA MARGARIDA LOSA | WWW.ILCML.COM
446 
${ }^{4}$ Vgl. das Stichwort “Agnes" in Seibicke, 1996: 44 u. die "etymologische Einleitung” in Voragine 1982: 86.

5 BEVNAT ist eine Vollerhebung, die auf dem amtlichen Register Infostar (Zentrales Register der Zivilstandsereignisse Geburt, Tod, Eheschliessung, Scheidung, Vaterschaftsanerkennung, Adoption und eingetragene Partnerschaft) des Eidgenössischen Amtes für das Zivilstandswesen (EAZW) basiert. Vgl. O. A., Erhebungen, Quellen: o. S.

${ }^{6}$ In einer anderen Arbeit wurde auf den Zusammenhang dieses Kapitels mit dem entstehungsgeschichtlich relevanten Ausflug Stamms mit seiner damaligen Freundin nach Fribourg verwiesen. Vgl. Jambor, 2012: 343. .

7 Tanja van Hoorn schreibt abschließend zu Agnes treffend: "Die schlacksig-spröde, nachdenklichdifferenzierte und schon immer etwas todessehnsüchtige Agnes wird dem blassen Sachbuchautor zur Fluchtmöglichkeit in fiktive Welten und verschwindet darüber. Zurück bleiben ein ratloser Ich-Erzähler und das schillernde Bild einer rätselhaften Frau" (van Hoorn 2010: 3). 